\title{
Effect of cervical pessary on pregnancy outcome in patients with twin pregnancies: a meta-analysis
}

\author{
Yue Wang ${ }^{1}$, Jiaxing $\mathrm{Wu}^{2}$, Di $\mathrm{Ma}^{1}$, and mingli Huang ${ }^{1}$ \\ ${ }^{1}$ First Affiliated Hospital of Harbin Medical University \\ ${ }^{2}$ Shanghai Jiaotong University School of Medicine Xinhua Hospital
}

October 25, 2021

\begin{abstract}
Background Preterm birth is the main cause of child death under 5years of age. The incidence of twin pregnancies is less than $2 \%$, but the incidence of preterm delivery is $50 \%$ and the risk of neonatal death is 5 times higher in twin pregnancies than in singleton pregnancies.However,there is still no consensus on the effect of cervical pessary on preventing preterm delivery, prolonging the pregnancy cycle, and improving maternal and infant outcomes in patients with twin pregnancies. Objectives To explore the effect of cervical pessary on the pregnancy outcome of unselected twin pregnancy patients. Search Strategy Up to July 2021,researchers searched PubMed, EMBASE, COCHRANE, Web of Science, Wanfang, Weipu, and CNKI databases for research. Selection Criteria Randomized controlled trials that compared cervical pessary with standard care (no pessary) or alternative interventions(conventional and standard treatment (e.g.Atoxiban therapy) or vaginal progesterone) in patients with twin pregnancies. Data Collection and Analysis Two authors(W-Y and M-D)independently extracted information related to the study characteristics and test results from each of the included literature, and used Revman 5.3 to analyze the data. Conclusion The cervical pessary can extend the gestational week of short-cervix twin pregnancy without clinical symptoms, reduce the premature birth rate before 34 weeks of gestation, improve pregnancy outcome, reduce neonatal mortality, reduce necrotizing enterocolitis incidence, neonatal sepsis incidence, and improve neonatal outcome.
\end{abstract}

Effect of cervical pessary on pregnancy outcome in patients with twin pregnancies: a meta-analysis

Wang yue ${ }^{1}, \mathrm{Wu}$ jiaxing ${ }^{2}, \mathrm{Ma} \mathrm{di}^{3}$ Huang mingli ${ }^{*},{ }^{1}$ Department of Obstetrics and Gynecology, First Affiliated Hospital of Harbin Medical University Harbin, Heilongjiang, CN ${ }^{2}$ Department of Obstetrics and Gynecology, Xinhua Hospital Affiliated to Shanghai Jiao Tong University School of Medicine, 1665 Kongjiang Road, Shanghai, ${ }^{3}$ Department of Obstetrics and Gynecology, First Affiliated Hospital of Harbin Medical University Harbin, Heilongjiang, CN,China.Medical University,Harbin China.

Effect of cervical pessary on pregnancy outcome in patients with twin pregnancies: a meta-analysis

[Abstract]

Background Preterm birth is the main cause of child death under 5years of age.The incidence of twin pregnancies is less than $2 \%$, but the incidence of preterm delivery is $50 \%$ and the risk of neonatal death is 5 times higher in twin pregnancies than in singleton pregnancies.However, there is still no consensus on the effect of cervical pessary on preventing preterm delivery, prolonging the pregnancy cycle, and improving maternal and infant outcomes in patients with twin pregnancies.

Objectives To explore the effect of cervical pessary on the pregnancy outcome of unselected twin pregnancy patients.

Search Strategy Up to July 2021,researchers searched PubMed, EMBASE, COCHRANE, Web of Science, Wanfang, Weipu, and CNKI databases for research. 
Selection Criteria Randomized controlled trials that compared cervical pessary with standard care (no pessary) or alternative interventions(conventional and standard treatment (e.g.Atoxiban therapy) or vaginal progesterone) in patients with twin pregnancies.

Data Collection and Analysis Two authors(W-Y and M-D)independently extracted information related to the study characteristics and test results from each of the included literature, and used Revman 5.3 to analyze the data.

Main Results The researchers included a total of 7 documents with a total of 3074 patients. Among them, 4 studies included pregnancy outcomes and neonatal outcomes for patients with cervix length $<25 \mathrm{~mm}$, and 7 studies included pregnancy outcomes and neonatal outcomes for patients with cervix length $<38 \mathrm{~mm}$. The main results were premature delivery at $<34$ weeks, preterm delivery $<37$ weeks, and miscarriage $<28$ weeks. Secondary results included spontaneous preterm delivery $<34$ weeks, spontaneous preterm delivery $<34$ weeks, spontaneous miscarriage $<28$ weeks, and preterm prelabour rupture of membranes $<34$ and preterm prelabour rupture of membranes, vaginal bleeding, chorioamnionitis, delivery week, vaginal infection, vaginal discharge, cesarean section, intrauterine death or stillbirth, neonatal death, low weight birth, very low weight birth, neonatal respiratory distress syndrome, neonatal intraventricular hemorrhage, necrotizing enterocolitis, retinopathy, sepsis. The results showed that the incidence of delivery week, vaginal discharge, and vaginal bleeding in the experimental group was higher than that in the control group, which was statistically significant. For neonates, the incidence of low-weight children, necrotizing enterocolitis and neonatal septicemia were significantly lower in the experimental group and were statistically significant. Subgroup analysis results based on cervical length $<38 \mathrm{~mm}$ showed that cervical pessary could reduce the preterm birth rate before 34 weeks, the spontaneous preterm birth rate before 34 weeks, prolong the gestational week of delivery, reduce neonatal mortality and the occurrence of neonatal necrotizing enterocolitis. The rate and incidence of neonatal sepsis were better than those of the control group. However, the incidence of events such as increased vaginal discharge and vaginal bleeding in the experimental group was significantly higher than that in the control group, and the results were statistically significant. The results of subgroup analysis based on the cervical length $<25 \mathrm{~mm}$ showed that cervical pessary was better than the control group in reducing the preterm birth rate before 34 weeks, the spontaneous preterm birth rate before $<34$ weeks, and the incidence of low-birth-weight infants, and the results were statistically significant.

Conclusion The cervical pessary can extend the gestational week of short-cervix twin pregnancy without clinical symptoms, reduce the premature birth rate before 34 weeks of gestation, improve pregnancy outcome, reduce neonatal mortality, reduce necrotizing enterocolitis incidence, neonatal sepsis incidence, and improve neonatal outcome. For patients with a cervical length less than $38 \mathrm{~mm}$, a cervical pessary can be performed to extend the gestational week. For patients with a cervical length less than $25 \mathrm{~mm}$, a cervical pessary can effectively prolong the gestational age and improve the maternal and fetal outcomes. The cervical pessary is safe for patients with twin pregnancies. In terms of long-term efficacy, there is no evidence of cervical support placement on the long-term maternal prognosis. In terms of economic benefits, cervical support is better than vaginal progesterone,but the conclusion still needs more research to prove.

Keywords: cervical pessary; pregnancy outcome; fetal outcome; preterm birth;

\section{Introduction}

Studies have shown that the mortality rate for children under 5 years of age in China is 37\%, with the main cause of death being complications from premature birth, accounting for about $17 \%$ of all deaths ${ }^{1}$.Surviving preterm infants are at greater risk for short-term complications, with higher rates of respiratory distress syndrome, bronchopulmonary dysplasia, necrotizing enterocolitis, sepsis, intraventricular hemorrhage, paraventricular leukodystrophy, and retinopathy than in term-born neonates ${ }^{2}{ }^{3}$. The incidence of twin pregnancies is less than $2 \%$, but the incidence of preterm delivery is $50 \%$ and the risk of neonatal death is 5 times higher in twin pregnancies than in singleton pregnancies ${ }^{45}$.Cervical insufficiency as a cause of spontaneous preterm delivery in patients with twin pregnancies ${ }^{6}$.

Currently, the main therapeutic measures regarding the prevention of preterm delivery in patients with twin 
pregnancies are the vaginal progesterone, cervical cerclage and cervical pessary.In the Clinical Guidelines for the Management of Twin Pregnancies published in China in 2020, it is clearly stated that in asymptomatic patients with twin pregnancies with a short cervix, the use of progestins can effectively reduce the risk of preterm delivery before 35 weeks of gestation ${ }^{7}$. As for cervical cerclage, for singleton patients, this treatment is currently considered effective in preventing preterm birth $^{8}$, but for patients with twin pregnancies, the efficacy of the treatment remains controversial ${ }^{7}$.

Originally used to treat pelvic organ prolapse, cervical pessary placement has been used to prevent preterm birth since 1990. Currently, the Arabin uterine support is widely used in the treatment of spontaneous preterm labor. It is designed with the intention not only to support and compress, but also to tilt the cervix and possibly rotate it towards the sacrum, mainly by supporting the inner cervical opening and preventing it from being overburdened with gravity ${ }^{10}$. Currently studies show that cervical pessary placement is effective in reducing the rate of preterm birth in patients with singleton pregnancies ${ }^{11}{ }^{12}$, while cervical pessary placement remains controversial in patients with twin pregnancies.A retrospective analysis in 2016 showed that cervical pessary placement reduced the rate of preterm delivery before 37 and 34 weeks of gestation in patients with twin pregnancies ${ }^{13}$.In 2019, an RCT trial suggested that cervical pessary placement reduces preterm birth rates and improves pregnancy outcomes in patients with twin pregnancies ${ }^{14}$,but There were RCT experiments and meta-analyses that came to the opposite conclusion ${ }^{15-17}$.

In conclusion, there is still no consensus on the effect of cervical pessary on preventing preterm delivery, prolonging the pregnancy cycle, and improving maternal and infant outcomes in patients with twin pregnancies. In this article, we present a meta-analysis of the effects of cervical pessary placement on pregnancy outcomes in patients with twin pregnancies and discuss the effectiveness of cervical pessary placement in improving adverse pregnancy outcomes and neonatal outcomes.

Materials and Methods

\subsection{Literature Search}

This study was conducted and reported in accordance with the Preferred Reporting Items for Systematic Reviews and Meta-Analyses (PRISMA) statement ${ }^{18}$, and was registered with PROSPERO, numberCRD42021275530.Two authors independently extracted all study data into a canonical form. When there is a difference of opinion, the two authors reach a consensus through negotiation.

Up to July 2021, researchers searched PubMed, EMBASE, COCHRANE, Web of Science, Wanfang, Wipu and CNKI databases, for example, in the PubMed database, they searched for "cervical pessary", "preterm birth ", "spontaneous preterm birth", "cervical insufficiency", "twin pregnancy", "RCT study " Investigators selected studies for inclusion that met the inclusion standards.Relevant publications were searched.Researchers also systematically reviewed the references of the literature included in the study.Clinical pregnancy outcomes and neonatal outcomes were collected in the cervical pessary group (experimental group) and the non-cervical pessary group (control group).Two reviewers reviewed each potential eligibility article separately, analyzed the quality of the studies according to the Cochrane scale, and extracted data.Two authors (W-Y and M-D)independently performed the original screening of all study titles and abstracts, excluding literature that was deemed irrelevant by both observers. The PRISMA flowchart provides more detailed information about the article selection process(figure 1: flowchart). The researchers recorded in detail the year of publication of the records, country, study type, number of participants, week

of gestation and range of cervical length (CL) at the time of placement of the cervical pessary, and mode of treatment for both groups of patients (table 1:characteristics of the included studies).

\subsection{Inclusion standards for the study}

The purpose of the included study was to investigate the effect of cervical pessary placement on pregnancy outcomes in patients with twin pregnancies. The inclusion criteria were:1.Patients with twin pregnancies who underwent gynecological trans-vaginal ultrasound after 16 weeks of gestation and underwent Arabin cervical pessary because of the short cervix. There were no special requirements for the mode of pregnancy 
(ART and non-ART), and medical history of patients with twin pregnancy), 2.Must be done for RCT studies, 3.The experimental group must be patients undergoing cervical pessary, which can be performed in combination with conventional and standard treatment (e.g.Atoxiban therapy) or vaginal progesterone, 4 . The control group must be routine noninvasive treatment such as conventional therapy (such as atoxeban) or vaginal progesterone. The exclusion criteria were: 1.patients had cervical cerclage, 2.patients who needed to receive fetoscopy, 3. Exclusion abnormal fetal development, pregnancies of three and more fetuses, medically indicated preterm birth of medical origin(Twin-twin transfusion syndrome, severe preeclampsia, placenta abruption, placenta praevia, prenatal bleeding); 4. Non-RCT experiments.

\subsection{Data item extraction and quality evaluation}

Two researchers extracted information related to the study characteristics and test results from each of the included literature.The main outcome was preterm delivery (ptd) before 34 weeks gestation,preterm delivery before 37 weeks, miscarriage a before 28 weeks. Secondary outcomes included spontaneous preterm delivery(sptd) before 34 weeks,spontaneous preterm delivery before 37 weeks,spontaneous miscarriage(sm) before 28 weeks,preterm prelabor rupture of membranes(PPROM),preterm prelabor rupture of membranes before 34 week, deliver week(DW), vaginal bleeding(VB), chorioamnionitis(C), vaginal infection(VI), vaginal discharge(VD), C-section, intrauterine death or stillbirth, neonatal death, low-birth weight $(<2500 \mathrm{~g})$, very low birth weight $(<1500 \mathrm{~g})$, Respiratory distress syndrome (RDS),Intraventricular hemorrhage(IVH),Necrotizing Enterocolitis (NE), Retinopathy (RE),Sepsis (S).

\subsection{Risk assessment of bias}

Cochrane collaborative tools were used to assess the risk of bias in individual studies,including: 1 .selection bias; 2.implement bias; 3.detection bias; 4.follow-up bias; 5.reporting bias; 6 . measuring bias; 7.sampling bias (See details in the table2:bias of included literature assessment table).

\subsection{Data processing and analysis}

Researchers used Revman 5.3 to analyze the data.It belonged to two categorical outcome variables and relative rate $(\mathrm{RR})$ was used as the effect indicator,For the outcome index belonging to the continuous variables, the standards mean difference (SMD) was used as the effect indicator.95\% confidence interval was calculated to evaluate the strength of the association between cervical trust placement and the risk of adverse pregnancy-related outcomes. The $\mathrm{RR}$ values were calculated by the $\mathrm{Z}$ test.The $\mathrm{P}$-value of $<0.05$ was defined as meaningful.Random-effects and fixed-effects models were applied in this meta-analysis.To assess inter-study heterogeneity, the Q test was applied to calculate $\mathrm{I}^{2}$.The $\mathrm{I}^{2}$ values were defined as $25 \%, 50 \%$, and $75 \%$, representing low, moderate, and high heterogeneity, respectively. When high heterogeneity was observed, random-effects models were used to pool results, and a fixed-effect model. When the heterogeneity is too high, there should be further excluded clinical and methodological heterogeneity, and a random effect model is used for analysis.If there was evidence of statistical heterogeneity

$\left(\mathrm{I}^{2}>50 \%\right)$, it is necessary to explore the possible sources by using sensitivity and subgroup analyses to search for evidence of bias or methodological differences among trials. Researchers used the exclusion method article by piece exclusion literature method for sensitivity analysis.Differences in the elimination results and the original merger results were also assessed.Publication bias was visually judged by drawing funnel plots.

Results

\subsection{Results of literature search}

A total of seven literature studies have included 3,07 4 patients, and four studies containing $<25 \mathrm{~mm}$ for CL.For patient pregnancy and neonatal outcomes, seven studies included pregnancy and neonatal outcomes for patients with CL $<38 \mathrm{~mm}$.

2.2 Primary and secondary outcome summary

2.2.1.A meta analysis was performed for all of the literature 
First, we performed meta analyses on all included literature to compare the efficacy of cervical support in improving pregnancy outcomes with neonatal outcomes (see table3: Summary Results 1 ),It was found that the patients in the experimental group (cervical pessary group) had a longer deliver week $\left(\mathrm{OR} \quad 6.82,95 \% \mathrm{CI} \quad(0.18,0.32), \mathrm{I}^{2}=81 \%, \mathrm{P}<0.00001\right)$, higher incidence of vaginal discharge(OR12.96,95\% CI $\left.(4.17,7.02), \mathrm{I}^{2}=97 \%, \mathrm{P}<0.00001\right)$ and vaginal bleeding $(\mathrm{OR}$ $\left.5.34,95 \% \mathrm{CI}(1.32,1.83), \mathrm{I}^{2}=93 \%, \mathrm{P}<0.0001\right)$ than in the control group, which was statistically significant;For neonates,in experimental groups, the incidence of low birth weight(OR 4.14, 95\% CI $(0.86,0.95)$, $\left.\mathrm{I}^{2}=93 \%, \mathrm{P}<0.00001\right), \quad \mathrm{NE}\left(\mathrm{OR} 2.45,95 \% \mathrm{CI}(0.36,0.89), \mathrm{I}^{2}=40 \%, \mathrm{P}=0.01\right)$ and neonatal septicemia(OR2.21, $\left.95 \% \mathrm{CI}(0.43,0.95), \mathrm{I}^{2}=55 \%, \mathrm{P}=0.03\right)$ were significantly lower than control group, which were statistically significant.Compared DW, vaginal discharge, vaginal bleeding,low birth weight,S,remarkable heterogeneity could be seen. The sensitivity analysis was performed article by article, and the exclusion literature name and the $\mathrm{p}$ values after analysis are shown in the figure below. The funnel map suggests publication bias in part of the study.

\subsubsection{Subgroup analysis was performed based on the CL}

During the process of literature inclusion, the researchers found differences in the CL of the included patients, and to avoid the outcome bias caused by different CL, the subgroup analysis was performed.

Results of the subgroup analysis based on CL $<38 \mathrm{~mm}$ rows are shown in table 4: summary results 2, It can be seen that cervical pessary was better than the control group of preventing spontaneous premature delivery before 34 weeks $\left(\mathrm{OR} 2.18,95 \% \mathrm{CI}(0.61,0.79), \mathrm{I}^{2}=61 \%, \mathrm{P}=0.03\right)$ and premature delivery before 34 weeks $\left(\mathrm{OR} 2.9,95 \% \mathrm{CI}(0.38,0.73), \mathrm{I}^{2}=43 \% \mathrm{P}=0.004\right)$, prolonged delivery week $(\mathrm{OR} 6.31,95 \% \mathrm{CI}$ $\left.(0.26,0.48), \mathrm{I}^{2}=83 \%, \mathrm{P}<0.00001\right)$,reducing neonatal mortality(OR2.47,95\% CI(0.29,0.87),I2=73\%,P0.01), neonatal necrotic enterocolitis(OR3.52, $\left.95 \% \mathrm{CI}(0.18,0.61), \mathrm{I}^{2}=0 \%, \mathrm{P}=0.0004\right)$ and neonatal sep$\operatorname{sis}\left(\mathrm{OR} 2.21,95 \% \mathrm{CI}(0.43,0.95), \mathrm{I}^{2}=55 \%, \mathrm{P}=0.03\right)$. All the results were statistically significant. However, the incidence of increased vaginal discharge and vaginal bleeding in the cervical pessary group was significantly higher than in the control group. Some studies funnel plots suggest publication bias.

Results of the subgroup analysis based on the $<25 \mathrm{~mm}$ line of CL are shown in table 5:summary results 3.Due to insufficient data, comparisons were made only between premature delivery before 34 weeks(OR3.82,95\% CI $\left.(0.38,0.73), \mathrm{I}^{2}=0 \%, \mathrm{P}=0.0001\right)$, spontaneous premature delivery before 34 weeks(OR2.7,95\% CI(0.54,0.98), $\left.\mathrm{I}^{2}=80 \%, \mathrm{P}=0.04\right)$, low weight $\operatorname{birth}\left(\mathrm{OR} 6.32,95 \% \mathrm{CI}(0.54,0.72), \mathrm{I}^{2}=95 \%, \mathrm{P}<0.00001\right)$ and vary low weight $\operatorname{birth}\left(\mathrm{OR} 0.16,95 \% \mathrm{CI}(0.68,1.38), \mathrm{I}^{2}=0 \%, \mathrm{P} 0.87\right)$. Results showed that cervical pessary was better in reducing spontaneous preterm birth $<34$ weeks and preterm birth $<34$,decreased the born of lower weight infants, and the results were statistically significant.Some study funnel plots suggested publication bias.

\subsection{Quality evaluation and bias evaluation}

These literatures were assessed for the risk of bias. Considering the particularity of the study, that the patients in the experiment knew all about their intervention after participating in the experiment, the researchers default that patients blindness are low-risk, and only evaluate the blind method of the researchers and the results assessors. Results showed that the implementation bias was low risk. One article had followup bias in the included literature. The rest of the bias was all low-risk (Figure 2:Risk bias assessment).

\section{Discussion}

\subsection{Main findings and data interpretation}

The results found that a general meta analysis of the efficacy of cervical support in twin patients only suggested that cervical support prolonged the gestational week of twin patients and reduced the incidence of low weight birth, NE and the incidence of neonatal sepsis. The $2012 \mathrm{CL}<25 \mathrm{~mm}$ can be short cervix considered for singleton guidelines published by American Obstetricians and Gynecologists (ACOG) ${ }^{19}$. However, there were also studies using a CL of less than the 25 th percentile $(<38 \mathrm{~mm})^{20} 21$.Therefore, the researchers used these two sets of digital row subgroup analysis, respectively.The results suggested that in twin patients with 
$<38 \mathrm{~mm}$ of CL, cervical pessary reduced preterm birth $<34$ weeks, decreased rate of spontaneous premature delivery $<34$ weeks, prolonged delivery week, reduced neonatal mortality,neonatal necrotizing enterocolitis, and neonatal sepsis; For patients with CL $<25 \mathrm{~mm}$, although the data are insufficient, limited data still suggest that cervical pessary is better than conventional treatment in reducing preterm birth rate $<34$ weeks, spontaneous preterm birth $<34$ weeks, and reducing the incidence of low-weight infants.Although, increased vaginal secretions and higher vaginal bleeding rate were higher in the experimental group when $<38 \mathrm{~mm}$ of the CL was observed, there was no significant difference in the incidence of vaginitis and chorioamnionitis compared with the control groups. This shows that cervical pessary can effectively reduce the rate of preterm birth before 34 weeks in twin patients with CL less than $38 \mathrm{~mm}$, effectively prolong pregnancy week, reduce neonatal mortality, neonatal necrotizing enterocolitis and neonatal sepsis, and effectively improve adverse maternal pregnancy outcome.

\subsection{Sensitivity analysis}

During the course of the sensitivity analysis, the researchers found the phenomena of altered $\mathrm{P}$ values after the sensitivity analysis,After analyzing the sources of heterogeneity one by one, the researchers thought that the reasons for the heterogeneity were: 1 . The data gap is too large; 2 . There is a publication bias. 3.there are other potential unmeasured confounders.such as cervical surgery history, miscarriage history,difference race, the time of pregnance and delivery,and ART history. As Kypros 2015, in Results summary Table 1.It accounted for $30.1 \%$ and $43.1 \%$ in the two analyses of $\operatorname{ptd}<34$ and $\operatorname{sptd}<34$, and the negative results in this literature greatly interfered with the statistics of $\mathrm{ptd}<34$ and $\operatorname{sptd}<34$ results, and after the sensitivity analysis, excluding Kypros 2015, the relatively evenly distributed data showed positive results contrary to the original results. And the data accounted for too large literature, its funnel map suggests a publication bias.As to potential unmeasured confounders,researchers found several factors could cause confounders such as cervical surgery history, miscarriage history, difference race, the time of pregnancy or delivery,ART history,time of placing or removing cervical pessary,vaginal progesterone.Particularly,Dang's research ${ }^{20}$ included patients used vaginal progesterone, which has caused unmeasured confounders, which has shown in our research(see table 3and table 4). Current, only one retrospective cohort study of twin pregnancies draw a conclusion that cervical pessary combined with vaginal progesterone could prolonged pregnancy and reduce risk of adverse neonatal outcomes ${ }^{22}$. There is one study showed that cervical pessary combined with vaginal progesterone could prolonged pregnancy, reduce prematurity rate and a low rate of perinatal complications ${ }^{23}$. A metaanalysis $^{24}$ and a RCT in 2016 showed that combined treatment did not decrease the risk of preterm birth compared with cervical pessary in singletons with short CL ${ }^{25}$. Therefore, more clinical trials are needed. Therefore, it is not sure if vaginal progesterone could enhance or weaken the influence of cervical pessary to twin pregnancy.

\subsection{Current researches and guidelines}

Twin gestation are different from singleton patients, because of their excessive uterine enlargement, intrauterine pressure increases too much, beyond the limit that the cervix can bear, easy to lead to cervical insufficiency, and then cause premature birth.The treatment to prevent premature birth is still controversial.Cervical pessary placement was originally used to treat pelvic organ prolapse, and was been used to prevent preterm birth since 1990. Currently, Arabin cervical pessary has been widely used in the treatment of spontaneous preterm birth. It is designed to not only support and compression, but also tilt the cervix and may rotate it toward the sacrum, mainly by bearing the inner cervix, preventing it from bearing excessive gravity burden ${ }^{10}$. The current study shows that cervical pessary placement can effectively reduce the rate of preterm birth in patients with singleton pregnancies ${ }^{11}$.

For twin patients, cervical pessary is still controversial. Leim2013 was the first to study the treatment effect of cervical support in twin pregnancy patients through RCT experiments, and proposed that cervical uterine care could not effectively prevent adverse perinatal outcome or premature birth in multiple pregnant women, but its subgroup analysis proved that cervical pessary of the cervix in twin patients can reduce adverse perinatal outcome of $<38 \mathrm{mmm}^{26}$. This provided later researchers with ideas to study the relationship between different CLs and the efficacy of cervical trust placement.In 2016, a Retrospective analysis revealed 
that cervical pessary placement reduced the rate of preterm delivery that occurred before 36 and 34 weeks ${ }^{13}$.

Contradictory findings also exist in the RCT study. Kypros believes that cervical pessary does not reduce the preterm rate in twin patients with cervical insufficiency ${ }^{15}$. However, the RCT experiment published by Goya in 2015 for the first time that cervical pessary reduced the rate of preterm delivery by 34 weeks in twin patients with a CL of $<25 \mathrm{~mm}^{16}$. In 2019 , an RCT experiment proposed that cervical trust placement reduced the rate of preterm birth and improved pregnancy outcomes in patients with twin gestation ${ }^{[14}$. In 2017, by comparing treated patients with cervical pessary, cervical ring ligation and vaginal progesterone, a meta analysis proposed that only vaginal progesterone extended the gestational week, but not statistical significance $^{27}$. The latest meta analysis in 2020 does not support the use of cervical uterine support to prevent premature birth or improve perinatal outcomes in twin short cervix and unselected twin pregnancies ${ }^{17}$. However, patients included in the study included singletons, including studies using non-Arabin cervical support, leading to a bias in their findings.

In terms of the long-term effects of cervical care, only Noor $\mathrm{E}^{28}$ followed up participants for up to four years based on the Leim study. The results showed that the intervention group ( $\mathrm{CL}<38 \mathrm{~mm})$, but no difference in abnormal growth and development outcomes between the two groups. However, there were a large number of missed visits in the study, so at present, there is still insufficient data to prove that cervical care can lead to long-term adverse outcomes in mothers.

Similarly, only one study ${ }^{29}$ has proposed that after comparing the efficacy and economic benefits of cervical support with vaginal progesterone, cervical care improved adverse pregnancy outcomes and reduced costs. The conclusion still requires sufficient data support.

Differences in the results of RCT experiments led to the wavering of guidelines. No specific and effective treatment exists to prevent preterm birth in the 2014 ACOG guidelines for twin women ${ }^{30}$. In 2019, the Canadian Association of Obstetricians and Gynecologists (SOGC) still believed that, even in twin patients with a short cervix, there was still no evidence that cervical pessary could effectively prevent premature birth $^{9}$. China updated its guidelines in 2020 and did not give clear recommendations on the use of cervical pessary to prevent premature birth in twin patients. However, Chinese guidelines clearly support that vaginal progesterone can reduce the preterm birth and neonatal prevalence before 35 weeks in pregnant women with asymptomatic ultrasound showing a short cervix ${ }^{7}$. As for cervical cerclage, as an invasive treatment, its efficacy varies due to its different timing and indications. In 2014, ACOG noted that existing data demonstrated that ring ligation actually significantly increased preterm birth rates in asymptomatic twin pregnancies with $\mathrm{CL}<25 \mathrm{~mm}^{30}$. The SOGC guidelines also indicate that ring ligation increases asymptomatic preterm rates in twin pregnancies with CL $<25 \mathrm{~mm}$, arguing that no physical evidence of physical examination only with ultrasound hints of cervical shortening or previous second trimester abortion supports cervical placement increases the risk of preterm pregnancy. However, emergency ring ligation may be potentially valuable in patients with $\mathrm{CL}<15 \mathrm{~mm}$ or cervical dilated $>1 \mathrm{~cm}^{9}$. Chinese guidelines give the same recommendations.

\subsection{Strengths and limitations}

Seven high-quality RCT studies were included with a total of 3074 patients who performed a subgroup analysis based on different CLs based on the overall meta analysis, The results proved that although cervical pessary increased vaginal discharge and vaginal bleeding rate, it can effectively reduce the preterm birth rate of twins before 34 weeks with CL less than $38 \mathrm{~mm}$, effectively prolong gestational week, reduce neonatal mortality, neonatal necrotic enterocolitis and neonatal sepsis, and effectively improve the adverse pregnancy outcome.

There are shortcomings in this paper.First, even if more than 3,000 patients were included, the results were still affected by insufficient sample size and uneven sample distribution, resulting in altered results after sensitivity analysis. Second, only one of the seven studies included was about the efficacy comparison of cervical trust placement and vaginal progesterone, which brought some bias to our study. At the same time, the researchers regretted that the inability to compare the treatment effect of cervical trust placement with vaginal progesterone to prevent premature birth in twin patients had failed.Furthermore, only one study 
included was from Asia, and researchers similarly regret the inability to perform a race-based subgroup analysis. Otherwise, The subjects were unselected twin pregnancies and had no clear requirements for the mode of pregnancy (ART or non-ART) or medical history (with a history of miscarriage, delivery and cervical surgery), which may bias the results.For this part of the patients, additional attention is needed. Alternatively, timing differences in the inclusion literature exist to the current lack of uniform guidelines. It is seen that most of the pessary were placed between $16-24$ weeks, but Merced2019 was at $24-34$ weeks. The reason for this difference is selection bias due to the different subjects selected each study.

\subsection{Implications for practice and research}

Despite the multiple regrets, our approach is scientific and the results are valid. This paper reverses the conclusion proposed in the previous meta analysis and guidelines that "cervical pessary cannot effectively prolong gestational weeks and prevent preterm birth in twin pregnancies", and proves the effectiveness of cervical trust placement in patients with twin pregnancies.

Conclusion

Cervical pessary can extend the gestational week of short-cervix twin pregnancy without clinical symptoms, reduce the premature birth rate before 34 weeks of gestation, improve pregnancy outcome, reduce neonatal mortality, reduce neonatal enterocolitis incidence, neonatal sepsis incidence, and improve neonatal outcome. For patients with a cervical length less than $38 \mathrm{~mm}$, cervical pessary can be performed to extend the gestational week. For patients with cervical length less than $25 \mathrm{~mm}$, cervical pessary can effectively prolong the gestational age and improve the maternal and fetal outcome. Cervical pessary is safe for patients with twin pregnancies.In terms of long-term efficacy, there is no evidence of cervical support placement on the long-term maternal prognosis.In terms of economic benefits, cervical support is better than vaginal progesterone,but the conclusion still needs more research to prove.

Acknowledgments

We are grateful to Mr.Wu, Mrs.Ma and Mrs Huang for their contribution to this article on data collecting,writing, and polishing this study.Mrs Wang approved this project and participated in all parts. Mrs Ma and Mrs Huang were helped in data collecting. Mr Wu polished this paper and provided several prescious advices about data analysis.Mrs Huang recheck this paper at last. This study is not sponsored by any individuals or organizations, and there are no conflicts of interest or ethical challenges.

\section{Reference}

1. Song P, Theodoratou E, Li X, et al. Causes of death in children younger than five years in China in 2015: an updated analysis. Journal of global health 2016;6(2):020802. doi: 10.7189/jogh.06.020802 [published Online First: 2016/12/29]

2. Lynch AM, Wagner BD, Hodges JK, et al. The relationship of the subtypes of preterm birth with retinopathy of prematurity.American journal of obstetrics and gynecology2017;217(3):354.e1-54.e8. doi: 10.1016/j.ajog.2017.05.029 [published Online First: 2017/05/27]

3. Catov JM, Scifres CM, Caritis SN, et al. Neonatal outcomes following preterm birth classified according to placental features. American journal of obstetrics and gynecology 2017;216(4):411.e1-11.e14. doi: 10.1016/j.ajog.2016.12.022 [published Online First: 2017/01/10]

4. Houlihan C, Poon LC, Ciarlo M, et al. Cervical cerclage for preterm birth prevention in twin gestation with short cervix: a retrospective cohort study. Ultrasound in obstetrics $\&$ gynecology : the official journal of the International Society of Ultrasound in Obstetrics and Gynecology 2016;48(6):752-56. doi: 10.1002/uog.15918 [published Online First: 2016/11/02]

5. Huang X, Saravelos SH, Li TC, et al. Cervical cerclage in twin pregnancy. Best practice 8 research Clinical obstetrics $\& 3$ gynaecology 2019;59:89-97. doi: 10.1016/j.bpobgyn.2019.06.001 [published Online First: $2019 / 07 / 25]$ 
6. Vogel JP, Chawanpaiboon S, Moller AB, et al. The global epidemiology of preterm birth. Best Pract Res Clin Obstet Gynaecol2018;52:3-12. doi: 10.1016/j.bpobgyn.2018.04.003 [published Online First: 2018/05/22]

7. Guidelines for clinical treatment of twin pregnancy (updated 2020). Chinese Journal of Perinatal Medicine 2020 (08): 505-16.

8. Berghella V, Rafael T, Szychowski J, et al. Cerclage for short cervix on ultrasonography in women with singleton gestations and previous preterm birth: a meta-analysis. Obstetrics and gynecology2011;117(3):66371. doi: 10.1097/AOG.0b013e31820ca847

9. Brown R, Gagnon R, Delisle MF. No. 373-Cervical Insufficiency and Cervical Cerclage. J Obstet Gynaecol Can 2019;41(2):233-47. doi: 10.1016/j.jogc.2018.08.009 [published Online First: 2019/01/15]

10. Arabin B, Alfirevic Z. Cervical pessaries for prevention of spontaneous preterm birth: past, present and future. Ultrasound Obstet Gynecol 2013;42(4):390-9. doi: 10.1002/uog.12540 [published Online First: 2013/06/19]

11. Pratcorona L, Goya M, Merced C, et al. Cervical pessary to reduce preterm birth $<34$ weeks of gestation after an episode of preterm labor and a short cervix: a randomized controlled trial.Am J Obstet Gynecol 2018;219(1):99 e1-99 e16. doi: 10.1016/j.ajog.2018.04.031 [published Online First: 2018/04/29]

12. Saccone G, Maruotti GM, Giudicepietro A, et al. Effect of Cervical Pessary on Spontaneous Preterm Birth in Women With Singleton Pregnancies and Short Cervical Length: A Randomized Clinical Trial. Jama2017;318(23):2317-24. doi: 10.1001/jama.2017.18956 [published Online First: 2017/12/21]

13. Di Tommaso M, Seravalli V, Arduino S, et al. Arabin cervical pessary to prevent preterm birth in twin pregnancies with short cervix. Journal of obstetrics and gynaecology : the journal of the Institute of Obstetrics and Gynaecology 2016;36(6):715-18. doi: 10.3109/01443615.2016.1148127 [published Online First: 2016/03/26]

14. Merced C, Goya M, Pratcorona L, et al. Cervical pessary for preventing preterm birth in twin pregnancies with maternal short cervix after an episode of threatened preterm labor: randomised controlled trial. Am J Obstet Gynecol 2019;221(1):55 e1-55 e14. doi: 10.1016/j.ajog.2019.02.035 [published Online First: 2019/03/04]

15. Nicolaides KH, Syngelaki A, Poon LC, et al. Cervical pessary placement for prevention of preterm birth in unselected twin pregnancies: a randomized controlled trial. Am J Obstet Gynecol2016;214(1):3.e1-9. doi: 10.1016/j.ajog.2015.08.051 [published Online First: 2015/09/01]

16. Goya M, de la Calle M, Pratcorona L, et al. Cervical pessary to prevent preterm birth in women with twin gestation and sonographic short cervix: a multicenter randomized controlled trial (PECEP-Twins). $A m$ J Obstet Gynecol 2016;214(2):145-52. doi: 10.1016/j.ajog.2015.11.012 [published Online First: 2015/12/03]

17. Conde-Agudelo A, Romero R, Nicolaides KH. Cervical pessary to prevent preterm birth in asymptomatic high-risk women: a systematic review and meta-analysis. Am J Obstet Gynecol2020;223(1):42-65.e2. doi: 10.1016/j.ajog.2019.12.266 [published Online First: 2020/02/07]

18. The PRISMA Statement for Reporting Systematic Reviews and Meta-Analyses of Studies That Evaluate Health Care Interventions: Explanation and Elaboration. Annals of Internal Medicine 2009

19. Practice bulletin no. 130: prediction and prevention of preterm birth. Obstet Gynecol 2012;120(4):96473. doi: 10.1097/AOG.0b013e3182723b1b [published Online First: 2012/09/22]

20. Dang VQ, Nguyen LK, Pham TD, et al. Pessary Compared With Vaginal Progesterone for the Prevention of Preterm Birth in Women With Twin Pregnancies and Cervical Length Less Than $38 \mathrm{~mm}$ : A Randomized Controlled Trial. Obstet Gynecol 2019;133(3):459-67. doi: 10.1097/aog.0000000000003136 [published Online First: 2019/02/12] 
21. Liem SM, Schuit E, van Pampus MG, et al. Cervical pessaries to prevent preterm birth in women with a multiple pregnancy: a per-protocol analysis of a randomized clinical trial. Acta Obstet Gynecol Scand 2016;95(4):444-51. doi: 10.1111/aogs.12849 [published Online First: 2016/01/15]

22. Fox NS, Gupta S, Lam-Rachlin J, et al. Cervical Pessary and Vaginal Progesterone in Twin Pregnancies With a Short Cervix. Obstet Gynecol 2016;127(4):625-30. doi: 10.1097/aog.0000000000001300 [published Online First: 2016/03/10]

23. Daskalakis G, Zacharakis D, Theodora M, et al. Safety and efficacy of the cervical pessary combined with vaginal progesterone for the prevention of spontaneous preterm birth. J Perinat Med2018;46(5):531-37. doi: 10.1515/jpm-2017-0009 [published Online First: 2017/10/22]

24. Liu J, Song G, Meng T, et al. Vaginal progesterone combined with cervical pessary in preventing preterm birth: a meta-analysis. J Matern Fetal Neonatal Med 2021;34(18):3050-56. doi: 10.1080/14767058.2019.1677596 [published Online First: 2019/10/18]

25. Karbasian N, Sheikh M, Pirjani R, et al. Combined treatment with cervical pessary and vaginal progesterone for the prevention of preterm birth: A randomized clinical trial. J Obstet Gynaecol Res2016;42(12):1673-79. doi: 10.1111/jog.13138 [published Online First: 2016/10/09]

26. Liem S, Schuit E, Hegeman M, et al. Cervical pessaries for prevention of preterm birth in women with a multiple pregnancy (ProTWIN): a multicentre, open-label randomised controlled trial.Lancet 2013;382(9901):1341-9. doi: 10.1016/S0140-6736(13)61408-7 [published Online First: 2013/08/09]

27. Jarde A, Lutsiv O, Park CK, et al. Preterm birth prevention in twin pregnancies with progesterone, pessary, or cerclage: a systematic review and meta-analysis. Bjog 2017;124(8):1163-73. doi: 10.1111/14710528.14513 [published Online First: 2017/02/09]

28. Simons NE, van de Beek C, van der Lee JH, et al. Child outcomes after placement of a cervical pessary in women with a multiple pregnancy: A 4-year follow-up of the ProTWIN trial. Acta Obstet Gynecol Scand 2019;98(10):1292-300. doi: 10.1111/aogs.13630 [published Online First: 2019/04/30]

29. Le KD, Nguyen LK, Nguyen LTM, et al. Cervical pessary vs vaginal progesterone for prevention of preterm birth in women with twin pregnancy and short cervix: economic analysis following randomized controlled trial. Ultrasound Obstet Gynecol 2020;55(3):339-47. doi: 10.1002/uog.20848 [published Online First: 2019/08/23]

30. ACOG Practice Bulletin No.142: Cerclage for the management of cervical insufficiency. Obstet Gynecol 2014;123(2 Pt 1):372-79. doi: 10.1097/01.AOG.0000443276.68274.cc [published Online First: 2014/01/24]

31. Alexander EK, Pearce EN, Brent GA, et al. 2017 Guidelines of the American Thyroid Association for the Diagnosis and Management of Thyroid Disease During Pregnancy and the Postpartum. Thyroid2017;27(3):315-89. doi: 10.1089/thy.2016.0457 [published Online First: 2017/01/07]

32. Nicolaides KH, Syngelaki A, Poon LC, et al. Cervical pessary placement for prevention of preterm birth in unselected twin pregnancies: a randomized controlled trial. Am J Obstet Gynecol2016;214(1):3 e1-9. doi: 10.1016/j.ajog.2015.08.051 [published Online First: 2015/09/01]

33. Merced C, Goya M, Pratcorona L, et al. Cervical pessary for preventing preterm birth in twin pregnancies with maternal short cervix after an episode of threatened preterm labor: randomised controlled trial. Am J Obstet Gynecol 2019;221(1):55.e1-55.e14. doi: 10.1016/j.ajog.2019.02.035 [published Online First: 2019/03/04]

34. Norman JE, Norrie J, MacLennan G, et al. The Arabin pessary to prevent preterm birth in women with a twin pregnancy and a short cervix: the STOPPIT 2 RCT. Health Technol Assess 2021;25(44):1-66. doi: 10.3310/hta25440 [published Online First: 2021/07/06]

Figure 1 :flowchart 


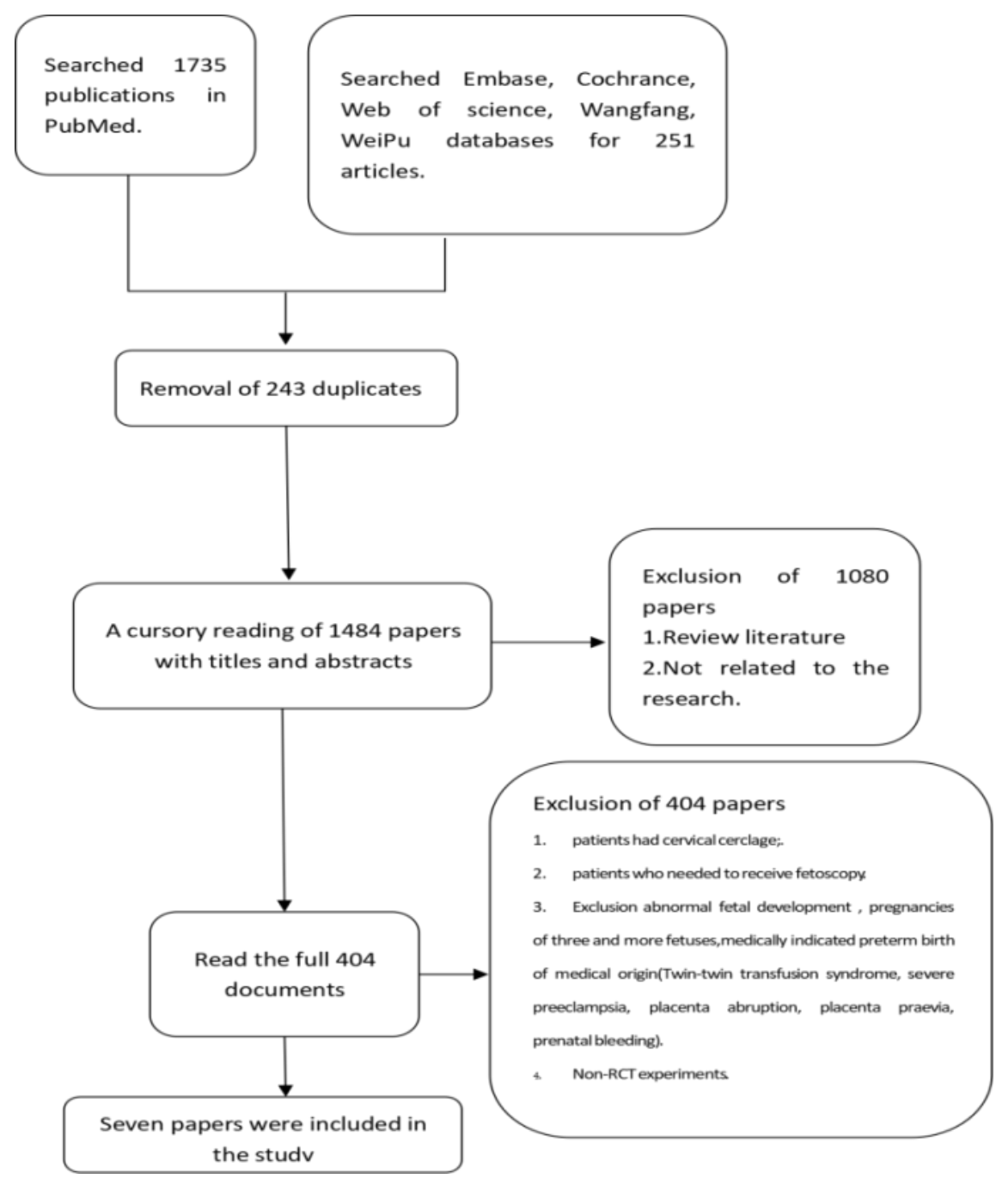

Table 1:characteristics of the included studies

\begin{tabular}{|c|c|c|c|c|c|}
\hline Name & Country & Type & Number & Characteristics & Treatment \\
\hline Berghella $2017^{31}$ & Multiple center & $\mathrm{RCT}$ & 46 & $\begin{array}{l}\text { 18-24week } \\
\mathrm{CL}<30 \mathrm{~mm}\end{array}$ & $\begin{array}{l}\text { Arabin Pessary } \\
\text { Conventional } \\
\text { treatment }\end{array}$ \\
\hline Goya $2015^{16}$ & Spain & $\mathrm{RCT}$ & 154 & $\begin{array}{l}18-22 \text { week } \\
\mathrm{CL}<25 \mathrm{~mm}\end{array}$ & $\begin{array}{l}\text { Arabin Pessary } \\
\text { Conventional } \\
\text { treatment }\end{array}$ \\
\hline $\begin{array}{l}\text { Nicolaided } \\
2015^{32}\end{array}$ & Multiple center & $\mathrm{RCT}$ & 1180 & $\begin{array}{l}\text { 18-25weel CL } \\
\text { unlimitated }\end{array}$ & $\begin{array}{l}\text { Arabin Pessary } \\
\text { Conventional } \\
\text { treatment }\end{array}$ \\
\hline Liem $2013^{26}$ & Netherlands & $\mathrm{RCT}$ & 808 & $\begin{array}{l}\text { 16-22week CL } \\
\text { unlimitated }\end{array}$ & $\begin{array}{l}\text { Arabin Pessary } \\
\text { Conventional } \\
\text { treatment }\end{array}$ \\
\hline Merced $2019^{33}$ & Spain & $\mathrm{RCT}$ & 132 & $\begin{array}{l}\text { 24-34week } \\
\mathrm{CL}<20 \mathrm{~mm}\end{array}$ & $\begin{array}{l}\text { Arabin Pessary } \\
\text { Conventional } \\
\text { treatment }\end{array}$ \\
\hline
\end{tabular}




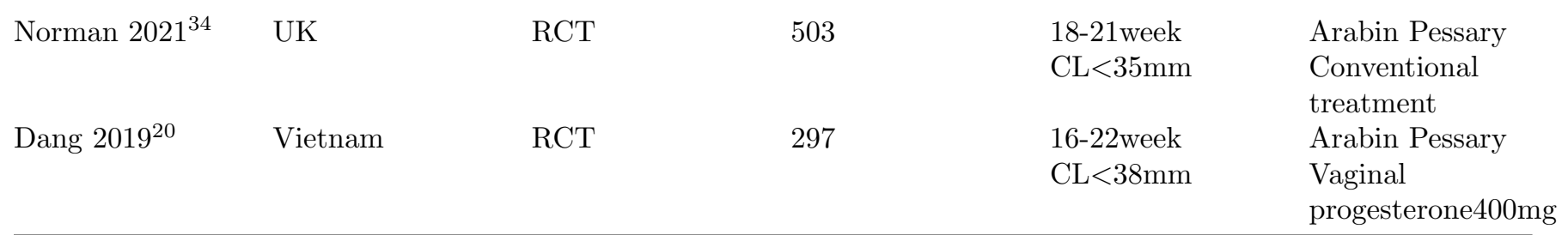

Table2 :the Bias of included Literature assessment table

\begin{tabular}{|c|c|c|c|c|c|c|c|}
\hline Name & selection bias & implement bias & detection bias & follow-up bias & reporting bias & $\begin{array}{l}\text { Other bias } \\
\text { measure bias }\end{array}$ & $\begin{array}{l}\text { Other } \\
\text { sampl }\end{array}$ \\
\hline Berghella2017 & - & - & - & - & - & - & - \\
\hline Goya2015 & - & - & - & - & - & - & - \\
\hline Nicolaided 2015 & - & - & - & - & - & - & - \\
\hline Liem2013 & - & - & - & - & - & - & - \\
\hline Merced2019 & + & - & - & + & - & - & - \\
\hline Norman2021 & - & - & - & - & - & - & - \\
\hline Dang 2019 & - & - & - & - & - & - & - \\
\hline
\end{tabular}

Table 3: smmary results 1

\begin{tabular}{lllllll}
\hline Results & $\mathrm{P}$ & $95 \% \mathrm{CI}$ & $\mathrm{I}^{2}$ & $\mathrm{RR}$ & Exclude the literature name & The $\mathrm{p}$ values were af \\
$\mathrm{ptd}<34$ & 0.07 & $0.72,1.01$ & $51 \%$ & 1.83 & Nicolaided 2015 & - $^{*}$ \\
ptd $<37$ & 0.18 & $0.88,1.02$ & $0 \%$ & 1.35 & - & - \\
miscarriage $<28$ & 0.76 & $0.69,1.31$ & $0 \%$ & 0.31 & - & - \\
sptd $<34$ & 0.06 & $0.68,1.01$ & $60 \%$ & 1.86 & Nicolaided 2015 & $0.003^{*}$ \\
sptd $<37$ & 0.34 & $0.8,1.08$ & $0 \%$ & 0.96 & - & - \\
sm $<28$ & 0.18 & $0.44,1.17$ & $0 \%$ & 1.34 & - & - \\
DW & $<0.00001^{*}$ & $0.18,0.32$ & $81 \%$ & 6.82 & Dang 2019 & $<0.0001^{*}$ \\
C & 0.87 & $0.64,1.71$ & $0 \%$ & 0.17 & - & - \\
VF & 0.13 & $0.9,2.34$ & $23 \%$ & 1.52 & - & - \\
VD & $<0.00001^{*}$ & $4.17,7.02$ & $97 \%$ & 12.69 & Merced2019 \\
$<34$ PPROM & 0.56 & $0.59,2.64$ & $66 \%$ & 0.58 & Goya2015 & $<0.0001^{*}$ \\
PPROM & 0.14 & $0.91,1.94$ & $63 \%$ & 1.49 & Norman2021 & 0.08 \\
C-section & 0.07 & $0.99,1.19$ & $8 \%$ & 1.84 & - & 0.62 \\
VB & $<0.0001^{*}$ & $1.32,1.83$ & $93 \%$ & 5.34 & Merced2019 & - \\
intrauterine death or stillbirth & 0.48 & $1.57,1.30$ & $0 \%$ & 0.71 & - & $<0.00001^{*}$ \\
neonatal death & 0.95 & $0.68,1.45$ & $0 \%$ & 0.06 & - & - \\
$<2500$ & $<0.00001^{*}$ & $0.86,0.95$ & $93 \%$ & 4.14 & Merced2019 & - \\
$<1500$ & 0.98 & $0.85,1.19$ & $0 \%$ & 0.03 & - & $0.03^{*}$ \\
NE & $0.01^{*}$ & $0.36,0.89$ & $40 \%$ & 2.45 & - & - \\
IVH & 0.55 & $0.73,1.79$ & $0 \%$ & 0.59 & - & - \\
RDS & 0.93 & $0.83,1.19$ & $28 \%$ & 0.08 & - & - \\
RE & 0.08 & $0.9,6.00$ & $40 \%$ & 1.73 & - & - \\
S & $0.03^{*}$ & $0.43,0.95$ & $55 \%$ & 2.21 & Norman2021 & - \\
\hline
\end{tabular}

(*It indicates that the statistical results were statistically significant)

(CL:CL,DW:deliver week, VI:vaginal infection,VD:vaginal discharge,VB:vaginal bleeding) 
Table 4: summary results 2

\begin{tabular}{lllllll}
\hline $\mathrm{CL}<38 \mathrm{~mm}$ & $\mathrm{P}$ & $95 \% \mathrm{CI}$ & $\mathrm{I}^{2}$ & $\mathrm{RR}$ & Exclude the literature name & The p values were aft \\
ptd $<34$ & $0.004^{*}$ & $0.38,0.73$ & $43 \%$ & 2.9 & - & - \\
ptd $<37$ & 0.06 & $0.84,1.01$ & $11 \%$ & 1.85 & - & - \\
miscarriage $<28$ & 0.21 & $0.5,1.16$ & $30 \%$ & 1.26 & - & - \\
sptd $<34$ & $0.03^{*}$ & $0.61,0.79$ & $61 \%$ & 2.18 & Nicolaided 2015 & $0.003^{*}$ \\
sptd $<37$ & 0.34 & $0.8,1.08$ & $0 \%$ & 0.96 & - & - \\
sm $<28$ & 0.18 & $0.44,1.17$ & $0 \%$ & 1.34 & - & - \\
DW & $<0.00001^{*}$ & $0.26,0.48$ & $83 \%$ & 6.31 & Norman 2021 & $<0.00001^{*}$ \\
C & 0.71 & $0.58,2.21$ & $0 \%$ & 0.37 & - & - \\
VI & 0.26 & $0.8,2.33$ & $38 \%$ & 1.14 & - & - \\
VD & $<0.00001^{*}$ & $2.51,4.11$ & $94 \%$ & 9.37 & Merced2019 \\
$<34$ PPROM & 0.56 & $0.59,2.64$ & $66 \%$ & 0.58 & Goya2015 & $<0.00001^{*}$ \\
PPROM & 0.07 & $0.96,2.9$ & $62 \%$ & 1.82 & Norman2021 \\
C-section & 0.93 & $0.9,1.12$ & $0 \%$ & 0.09 & - & 0.08 \\
VB & $<0.00001^{*}$ & $1.32,1.83$ & $93 \%$ & 5.34 & Norman2021 & 0.69 \\
intrauterine death or stillbirth & 0.18 & $0.86,2.33$ & $0 \%$ & 1.36 & - & - \\
neonatal death & $0.01^{*}$ & $0.29,0.87$ & $73 \%$ & 2.47 & Leim2013 & $<0.00001$ \\
$<2500$ & 0.00001 & $0.67,0.81$ & $90 \%$ & 6.02 & Dang 2019 & - \\
$<1500$ & 0.9 & $0.77,1.34$ & $0 \%$ & 0.13 & - & 0.99 \\
NE & $0.0004^{*}$ & $0.18,0.61$ & $0 \%$ & 3.52 & - & 0.11 \\
IVH & 0.5 & $0.41,1.55$ & $41 \%$ & 0.68 & - & - \\
RDS & 0.18 & $0.6,1.1$ & $11 \%$ & 1.34 & - & - \\
S & $0.03^{*}$ & $0.43,0.95$ & $55 \%$ & 2.21 & Dang 2019 & - \\
\hline
\end{tabular}

(*It indicates that the statistical results were statistically significant)

(CL:CL,DW:deliver week, VI:vaginal infection,VD:vaginal discharge,VB:vaginal bleeding)

Table 5 :summary results 3

\begin{tabular}{lllllll}
\hline $\mathrm{CL}<25 \mathrm{~mm}$ & $\mathrm{P}$ & $95 \% \mathrm{CI}$ & $\mathrm{I}^{2}$ & $\mathrm{RR}$ & Exclude the literature name & The p values were after the sensitivity ar \\
$\mathrm{sptd}<34$ & $0.04^{*}$ & $0.54,0.98$ & $80 \%$ & 2.7 & Nicolaided 2015 & $0.0005^{*}$ \\
$\mathrm{ptd}<34$ & $0.0001^{*}$ & $0.38,0.73$ & $0 \%$ & 3.82 & - & - \\
$<2500$ & $<0.00001^{*}$ & $0.54,0.72$ & $95 \%$ & 6.32 & Merced2019 & $0.00001^{*}$ \\
$<1500$ & 0.87 & $0.68,1.38$ & 0 & 0.16 & - & - \\
\hline
\end{tabular}

(*It indicates that the statistical results were statistically significant)

(CL:CL,DW:deliver week, VI:vaginal infection,VD:vaginal discharge,VB:vaginal bleeding)

Figure 2: Risk bias assessment

\section{Hosted file}

image2.emf available at https://authorea.com/users/442762/articles/542943-effect-ofcervical-pessary-on-pregnancy-outcome-in-patients-with-twin-pregnancies-a-meta-analysis 